\title{
Lecture
}

\section{On the Origins of Diversity in Social Behavior}

\author{
LARRY J. YOUNG ${ }^{12)}$ and QI ZHANG ${ }^{1)}$
}

\begin{abstract}
Here we discuss the origins of diversity in social behavior by highlighting research using the socially monogamous prairie vole. Prairie voles display a rich social behavioral repertoire involving pair bonding and consoling behavior that are not observed in typical laboratory species. Oxytocin and vasopressin play critical roles in regulating pair bonding and consoling behavior. Oxytocin and vasopressin receptors show remarkable diversity in expression patterns both between and within species. Receptor expression patterns are associated with species differences in social behaviors. Variations in receptor genes have been linked to individual variation in expression patterns. We propose that "evolvability" in the oxytocin and vasopressin receptor genes allows for the repurposing of ancient maternal and territorial circuits to give rise to novel social behaviors such as pair bonding, consoling and selective aggression. We further propose that the evolvability of these receptor genes is due to their transcriptional sensitivity to genomic variation. This model provides a foundation for investigating the molecular mechanisms giving rise to the remarkable diversity in social behaviors found in vertebrates.
\end{abstract}

Key words : social behavior, oxytocin, social bonding, evolvability, vasopressin

\section{Introduction}

The diversity among animals in terms of body plan, physiology and behavior is astonishing and has kindled the imagination of countless students and scientists in fields ranging from evolutionary biology and genetics to psychology. The origins of that

1) Center for Social Neural Networks, Faculty of Humans Sciences, University of Tsukuba, Tsukuba Japan

1-1-1 Tennodai Research Building D Center for Social Neural Networks University of Tsukuba Tsukuba, Ibaraki 305-857 Japan

2 ) Center for Translational Social Neuroscience, Department of Psychiatry and Behavioral Sciences, Yerkes National Primate Research Center, Emory University, Atlanta GA, USA 954 Gatewood Rd. Yerkes National Primate Research Center Emory University Atlanta, GA 30329 USA

Corresponding author: LARRY J. YOUNG

E-mail: lyoun03@emory.edu

J-STAGE Advance Published Date: May 17, 2021. doi: 10.2502/janip.71.1.4 diversity amid the confines of rigid biological principles is one of the greatest mysteries of life. This diversity must ultimately originate from variation in the sequence of the As, Gs, $\mathrm{Cs}$ and $\mathrm{Ts}$ that make up the genome. A major goal of biology is to understand that relationships of the letters, words, and phrases of the genome to the poetry that is the organism. As behavioral neuroscientists, we are particularly interested in how genes organize brain circuitry and neurochemistry to give rise to innate behaviors that are essential for survival and reproduction, including sociality, sexuality, mating strategies, parental care and aggression. Social behaviors can be remarkably diverse even among closely related species. Evolution increases diversity in social behavior through selection for traits that maximize fitness in the context of environmental factors such as food/resource availability and predation. Modern neurobiology is providing unprecedented clues into the gene-brain-behavior mechanisms controlling social behaviors. 
Much of today's biology is predicated on the premise that biological mechanisms are fairly-well conserved across vertebrates. This assumption is important because this means that by studying animals, we can gain insights into general principles of human biology, and those principles may be useful for improving the human condition. Thus, studies in fish can yield insights into human brain development. Lizards are useful for examining the flexible nature of sexuality, including homosexuality (Young \& Alexander, 2012; Young \& Crews, 1995). Mice and rats are the most well studied laboratory species and provide insights into addiction, parental care, and a host of psychiatric and neurological disorders. Most neuroscientists working with rodent models hope that the information gained in their species will apply to humans as well. Both scientist and funding agencies hope for conservation in mechanisms across species to increase the validity of findings in animal models for the human condition (Young, Pitkow, \& Ferguson, 2002).

In recent years, there have been increasing investigations into the neuroendocrine, genetic and neurobiological mechanisms regulating social behaviors. Most of this work is now performed in laboratory mice to take advantage of the powerful genetic manipulations possible in this species. Beyond traditional gene knockouts, mice strains are now available that express specific transgenes, such as Cre recombinase, in specific cell types in the brain. When combined with viral vector approaches, these mouse lines give investigators an unprecedented ability to monitor brain circuit activity to gain insights in the circuit bases of behaviors. Such studies are now revealing detailed circuit mechanisms involved in social reward (Dolen, Darvishzadeh, Huang, \& Malenka, 2013; Nardou et al., 2019), parental care (Lischinsky \& Lin, 2020) and aggression (Lischinsky \& Lin, 2020) that undoubtedly have implications for our own species.

However, any human or animal psychologist knows that mice are not small, furry humans. Mice are unlikely to be useful for identifying mechanisms involved in emotions and behaviors that are so important for our species, including love, jealousy, empathy and compassion. Nor are mice representative of the incredible diversity in social behaviors observed among vertebrates in nature. A major challenge of behavioral neuroscience and animal psychology is to be able to exploit the genetic and neurobiological toolkit commonly used with mice in different model species to expand the scope of our understanding of behavioral diversity (Blumstein et al., 2010; Boender \& Young, 2020; Hofmann et al., 2014; Johnson \& Young, 2018). Not only will such an approach bolster our understanding of mechanisms relevant to human behavior, but it may also help us understand the nature of diversity in social behavior and psychology among humans. Thus, the diversity in social behaviors across species may ultimately be the key to translational research to improve treatments for psychiatric disorders (Ford \& Young, 2020).

\section{The prairie vole model}

One model species where the aforementioned challenge is being met is the socially monogamous prairie vole (Microtus ochrogaster) (Figure 1) (McGraw \& Young, 2010). Prairie voles are highly social rodents that form enduring pair bonds, typically mate for

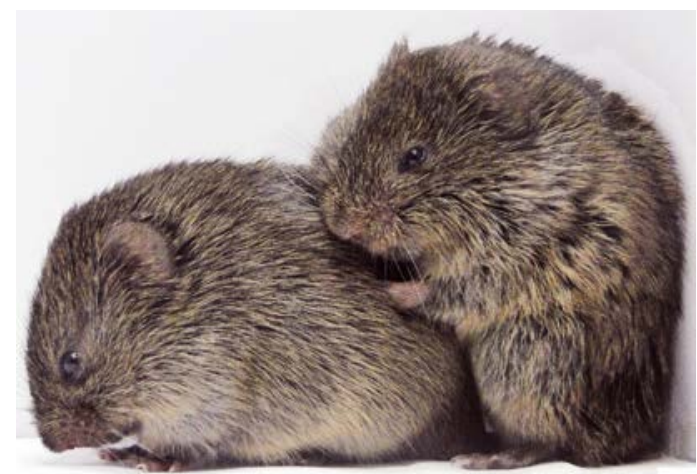

Figure 1. Photograph of a pair bonded couple of prairie voles (Microtus ochrogaster) 
life, and display biparental care. Like humans, not all prairie voles form monogamous bonds, and those that do occasionally exhibit extra-pair copulations (Okhovat, Berrio, Wallace, Ophir, \& Phelps, 2015). Nearly half of male prairie voles in nature remain as bachelors, mating opportunistically, sometimes with pair bonded females. Pair bonded prairie voles that lose their partner show behaviors reminiscent of grieving, or social loss-induced depression (Bosch et al., 2016; Bosch, Nair, Ahern, Neumann, \& Young, 2009; Bosch, Pohl, Neumann, \& Young, 2018; Bosch \& Young, 2018; Pohl, Young, \& Bosch, 2019). Prairie voles also display empathy-based consoling behavior toward their partner and other familiar prairie voles (Burkett et al., 2016). There are many species of voles in the Microtus genus that have similar appearance and genetic sequence, yet differ remarkably in social behavior (Fink, Excoffier, \& Heckel, 2006). For example, meadow voles (M. pennsylvani$c u s)$, which evolved under different evolutionary pressures, are relatively asocial, do not form pair bonds or display consoling behavior (Burkett et al., 2016; Walum \& Young, 2018; Young \& Wang, 2004). Both species have been studied extensively in the laboratory and have provided insights into the neural mechanisms giving rise to diversity in social behaviors (Donaldson \& Young, 2008). Furthermore, it is likely that the brain mechanisms giving rise to pair bonding, social loss-induced depressive behavior and consoling share deep homology with those mechanisms governing love, grief and compassion in humans (Young, 2009).

\section{Oxytocin, Vasopressin and Pair bonding}

Oxytocin (OXT) and arginine vasopressin (AVP) play important roles in the species differences in pair bonding behaviors in voles. OXT and AVP are peptide hormones synthesized in the hypothalamus and secreted into the bloodstream from the posterior pituitary where they regulate peripheral physiology (Johnson \& Young, 2017). Peripheral OXT regulates uterine contractions during birth and milk ejection during lactation. Plasma AVP regulates vascular tone and water balance (osmolarity). Both hormones also act in the brain to influence social behaviors. The OXT neurons projecting to the pituitary also project in the brain and when release in the CNS of mothers facilitate maternal nurturing behaviors and motherinfant bonding (Froemke \& Young, 2021; Ross, Cole, et al., 2009; Ross \& Young, 2009). AVP neurons projecting to the brain are sexually dimorphic and testosterone dependent and AVP regulates territorial behaviors (e.g. scent marking) and aggression, particularly in males (Albers, 2012). OXT signals in the brain via the OXT receptor (OXTR) while AVP signals primarily via the V1a AVP receptor (AVPR1A) (Donaldson \& Young, 2008). Evolutionarily, the OXT and AVP genes $(O x t, A v p)$ are believed to be derived from a duplication of a single ancestral peptide gene, and the OXTR and AVPR1A genes (Oxtr, Avpr1a) evolved and diverged in a similar manner (Grinevich, KnoblochBollmann, Eliava, Busnelli, \& Chini, 2016). Therefore, the OXT and AVP genes and their receptors genes are "cousins," genetically speaking, and therefore likely share genetic regulatory characteristics.

In prairie voles, OXT facilitates pair bond formation in both males and females. Blocking OXTR prevents mating-induced partner preferences, a laboratory proxy for pair bonds, in both sexes (Johnson et al., 2016; Young, Lim, Gingrich, \& Insel, 2001). Blocking AVPR1A inhibits mating-induced pair bonds and selective aggression, akin to mate-guarding, toward non-mates (Donaldson, Spiegel, \& Young, 2010; Gobrogge, Liu, Young, \& Wang, 2009; Nair \& Young, 2006). These results raised the question of why prairie voles form bonds, but meadow voles do not, from a neurobiological perspective. The density and distribution of OXT and AVP cells and fibers do not differ significantly between these species. By contrast, there are remarkable species differences in the distribution of OXTR and AVPR1A in the brain (Figure 2) (Lim, 


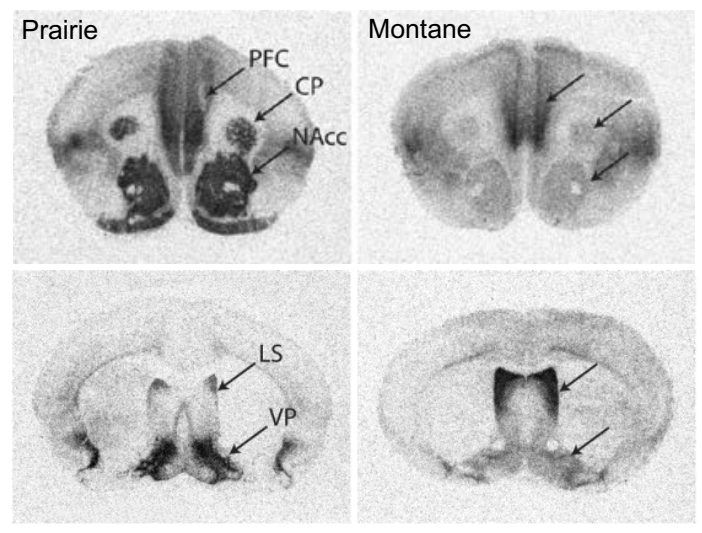

Figure 2. Receptor autoradiography illustrating species differences in oxytocin receptor (top) and vasopressin AVPR1A binding in monogamous prairie voles (left) and promiscuous montane voles (right). PFC, prefrontal cortex; $\mathrm{CP}$, caudate putamen; NAcc, nucleus accumbens; LS, lateral septum; VP, ventral pallidum

Murphy, \& Young, 2004; Young, Murphy Young, \& Hammock, 2005). In monogamous prairie voles, OXTR is concentrated in the nucleus accumbens (NAc), prefrontal cortex (PFC) and anterior cingulate cortex (ACC), but are absent or much less dense in these areas of promiscuous montane voles. Likewise, AVPR1A is highly expressed in the ventral pallidum (VP) in prairie voles but not in montane or meadow voles. Receptors are expressed in several areas in both species, including the amygdala and lateral septum.

This remarkable species difference in brain distribution is a hallmark feature of the OXTR and AVPR1A systems (Young, 1999). Indeed, rats mice and vole each have unique distributions of both receptors in the brain (Froemke \& Young, 2021). Similar species differences are also found among primates (Freeman \& Young, 2016; Rogers Flattery et al., 2021). In birds, species differences in OXTR are associated with flocking preferences in estrildid finches (Goodson, Schrock, Klatt, Kabelik, \& Kingsbury, 2009). This species diversity in neuropeptide distribution is in stark contrast to other receptor systems, including steroid receptors and dopamine receptors, whose distribution is remarkably conserved across species (King
\& Young, 2016).

There is good evidence that the species differences in receptor distribution contribute to species differences in vole social behaviors. Transgenic mice expressing the Avpr1 gene in a prairie vole distribution show elevated affiliative behavior following AVP administration while wildtype mice do not (Young, Nilsen, Waymire, MacGregor, \& Insel, 1999). Blocking OXTR signaling using antagonist or RNAi in the NAc or PFC prevents pair bonding in female prairie voles (Keebaugh, Barrett, Laprairie, Jenkins, \& Young, 2015; Walum \& Young, 2018; Young et al., 2001), while blocking AVPR1A in the VP inhibits partner preferences (Lim \& Young, 2004; Nair \& Young, 2006), but not mating, in male prairie voles. OXT and AVP signaling in other areas such as the lateral septum and anterior hypothalamus are also important for pair bonding and selective aggression in prairie voles (Gobrogge et al., 2009; Young \& Wang, 2004), but the NAc and VP are particularly interesting because of their role in reinforcement learning and addiction. The most compelling evidence that species differences in receptor distribution contribute to variation in social behavior comes from a study in which viral vectors were used to express the prairie vole Avprla in the VP of male meadow voles. Those meadow voles with AVPR1A expressed in the VP displayed partner preferences (Lim, Wang, et al., 2004). This result suggests that the species have similar neural circuitry but differ in how that circuitry is modulated by AVP. Thus, evolutionary plasticity in the expression pattern of Oxtr and Avprla likely mediate diversity in mating strategies in voles. Interestingly, monogamous marmosets and titi monkeys have OXTR/AVPR1A in NAc and VP, while non-monogamous rhesus macaques do not (Freeman, Inoue, Smith, Goodman, \& Young, 2014; Freeman, Walum, et al., 2014). OXTR mRNA is also highly expressed in the human NAc, although there is considerable variation in expression across individuals (Bethlehem et al., 2017). However, receptor expression in these areas do not 
predict mating strategy in monogamous and polygamous lemur species, suggesting that there is not a single neuropeptide modulated circuit that can give rise to monogamous behaviors across all species (Grebe et al., 2021).

The initial comparisons of vole species with diverse social behaviors highlighted the importance of OXTR signaling in the NAc for social bonding and subsequent complementary studies in voles and mice have been useful for constructing a model for how a simple peptide molecule can lead to a pair bond (Walum \& Young, 2018). Oxtr knockout mice display social amnesia and fail to recognize previously encountered mice. Social recognition in rodents is based primarily on olfactory cues. OT acts in both the anterior olfactory nucleus (AON) and the medial amygdala to facilitate social recognition (Ferguson, Aldag, Insel, \& Young, 2001; Oettl et al., 2016). OXTR signaling on AON projections to the olfactory bulb excites inhibitory granuale cells, which then inhibit the mitral output cells, to effectively inhibit "noise" activity. Thus, OXTR increases the signal to noise ratio. Similarly, OXTR enhances the salience of pup vocalizations in the auditory cortex by modulating excitatory/ inhibitory balance (Froemke \& Young, 2021). These examples suggest that OXTR signaling enhances the processing of salient social signals while inhibiting noise, increasing the quality of social cue information. This process is analogous to turning a dial on a television screen to minimize static, allowing the clear image to emerge. This high quality, high resolution olfactory signal can then be transmitted to the amygdala to facilitate social memory and attach valence. The signal, which encodes identity, can then be further transmitted to other brain regions in a social salience network, including the ventral hippocampus (social memory), PFC, NAc and ACC where the partner identity gains other attributes (e.g. reward, affect detection) (Walum \& Young, 2018). Studies in voles suggests that OXTR signaling facilitates communication across the social salience network, enhancing the flow of high resolutions social information across the network. By this process, OXTR signaling enhances the salience and reinforcing value of social cues, which has tremendous implications for treating social deficits in ASD (DeMayo, Young, Hickie, Song, \& Guastella, 2019).

\section{Partner Loss and "grieving"}

Once pair bonds are formed, partnership may be maintained not only by the reinforcing value of the partner, but also by the negative affect resulting from separation which drives enduring association (Bosch \& Young, 2018; Pohl et al., 2019). Prairie voles that have been separated from their partner for several days will show elevated stress axis activation and increase passive coping behavior, a depression-like behavior, and analogous to grieving. These responses are not observed in animals separated from their sibling and are therefore specific for separation from a bonded partner, perhaps akin to lovesickness in humans. Pair bonding results in increased synthesis of corticotrophin releasing factor (CRF) and separation from the partner may result in elevated CRF release. Inhibiting $\mathrm{CRF}$ receptor activation prevents partner loss-induced depression (Bosch et al., 2009). CRFR2 receptors are concentrated on hypothalamic OXT neurons projecting to the NAc, and CRFR2 agonist suppresses, while antagonist enhances, OXT release in the NAc (Bosch et al., 2016). Thus, it appears as if the withdrawal of OXTR signaling in the NAc may precipitate the grieving-like response following partner loss. Together, the work in voles suggest that because of the specific location of OXTRs in the brain, pair bonding results from the linking of the neural encoding of the partner to the NAc reward system, resulting in a sort of "addiction" to the partner, and loss of the partner suppresses OXTR signaling which leads to partner "withdrawal" and partner seeking behavior. Thus, a pair bond is in essence an addition to the partner, and loss of the partner can lead to physiological and 
psychological catastrophe (Burkett \& Young, 2012). This "yin and yang" action of OXT may be critical for the long-term maintenance of the pair bond. Although this model is still somewhat speculative, such a model could never have been constructed based on studies in mice alone. Interestingly, in pair bonded men, intranasal OXT enhances NAc BOLD signal while view images of their partner as well as subjective attractiveness of the partner, but not in response to other women, suggesting a common mechanism of oxytocin action in voles and man (Scheele et al., 2013).

\section{Empathetic Consoling}

Prairie voles have also provided insights into the neurobiological mechanisms underlying empathy. Both male and female prairie voles display a marked increase in partnerdirected grooming toward their distressed partner. This response has been referred to as consoling behavior, defined as an increase in affiliative contact in response to and directed toward a distressed individual which produces a calming effect (Burkett et al., 2016). This consoling behavior decreases stress-induced anxiety behaviors in the partner. Meadow voles do not display consoling behavior. The observers also display increased anxiety and fear behaviors, and elevated corticosterone levels that are correlated with those of the distressed partner, consistent with emotional contagion and physiological state-matching. Consoling behavior was also displayed toward siblings and unrelated cage mates, but not toward unfamiliar voles, demonstrating a familiarity bias. Elevated Fos protein, a marker of neural activity, was detected in the anterior cingulate cortex (ACC) following observing their distressed partner, and infusion of OXTR antagonist in the ACC of males eliminated consoling behavior. As the OXTR has been associated with empathy, emotion recognition and socioemotional engagement while the ACC has been linked to empathy in humans, this work suggests deep homology of underlying neural substrates between consoling behavior in voles and empathy in humans. Given OXT's role in maternal nurturing, it also suggests that derived behaviors, such as consoling behavior, involve tweaking of neural mechanisms of maternal care, which is ubiquitous in mammals, to reorient emotion detecting and nurturing response toward adult conspecifics (Burkett et al., 2016). Thus, consoling in voles may be an evolutionary antecedent of compassion, and an example of behavioral parallel evolution.

\section{Gene and circuit manipulations in voles.}

Mice have emerged as the dominant animal model in neuroscience primarily due to the ability to manipulate their genome, and consequently interrogate specific neural circuit function with cell-type specificity. Homologous recombination in stem cells allows for the ablation of genes, the development of conditional gene knockouts, and the insertion of Cre recombinase in tandem with genes of interest to activate an array of genetic tools for cell-type specific circuit manipulations. A major challenge for investigators working with non-traditional animal models has been the limited ability for genetic manipulations. Prairie voles have proven adaptable to genetic manipulations techniques, particularly with the development of viral vectors and Clustered Regularly Interspaced Short Palindromic Repeats (CRISPR) approaches. Early studies used adenoassociated viral vectors (AAV) to over-express Avprla or Oxtr to demonstrate that species differences or individual variation in receptor expression could contribute to diversity in social behavior in voles (Keebaugh \& Young, 2011; Lim, Wang, et al., 2004; Pitkow et al., 2001; Ross, Freeman, et al., 2009). AAV mediated RNA interference subsequently provided further evidence that individual variation in Oxtr expression in the NAc (Bosch et al., 2016; Keebaugh et al., 2015), or Avprla expression in the VP (Barrett et al., 2013), have functional influences on pair bonding related behaviors. More recently, viral vector approaches have enabled the manipulation of 
corticostriatal circuits to facilitate pair bonding using optogenetics (Amadei et al., 2017), and to identify the neural signature of monogamous reunion in the NAc using endoscopic GcAMP calcium imaging (Scribner et al., 2020). Finally, AAV-mediated CRISPR editing approaches have been successfully used to mutate Oxtr in adult vole brain with high efficiency (Boender \& Young, 2020).

While viral mediated gene transfer has been a fruitful approach, this strategy does not generate germline transgenic animals, which have been so useful in mice. The first germline transgenic vole was created using lentiviral mediated gene transfer to vole embryos, creating fluorescent green prairie voles (Donaldson, Yang, Chan, \& Young, 2009). However, this technique was relatively inefficient and has not been successful for more useful transgenes. More recently, Oxtr knockout and Oxtr-Cre prairie voles have been generated using CRISPR mediated embryo genome editing (Horie, Inoue, Nishimori, \& Young, 2020; Horie et al., 2019). The Oxtr-Cre transgenic voles provide a means of mapping Oxtr neuronal circuits, opto- or chemogenetic manipulations of specific circuits, and cell-type specific activity monitoring. As CRISPR editing is very efficient, we anticipate the development of many mutant voles that will greatly enhance the power of this model organism for investigating the neural circuit mechanism of social behaviors such as pair bonding, parternal care, and empathy.

\section{Evolvability of Avpr1a and Oxtr generate diversity in social behavior}

One principle that has emerged from studying social behavior in voles with a comparative perspective is that Avprla and Oxtr display remarkable phylogenetic plasticity and even individual variation that appears to be linked to diversity in social behaviors (Donaldson \& Young, 2008; King \& Young, 2016; Young, 1999). In other words, both Avpr1a and Oxtr display a high degree of "evolvability," defined as the ability of a biological system to produce phenotypic variation that is both heritable and adaptive (Payne \& Wagner, 2019). We hypothesize that this evolvability in brain distribution is a transcriptional regulatory trait shared by Avpr1a and Oxtr due to their common ancestry and subsequent divergence following gene duplication. We propose that the mechanisms underlying this transcriptional evolvability may represent the key driving force generating diversity in social behavior among vertebrates.

There are remarkable species differences in both Avprla and Oxtr expression in the brains of mammals, including primates, that likely contribute to species differences in social behavior (Donaldson \& Young, 2008; King \& Young, 2016; Rogers Flattery et al., 2021; Young, 1999). Even among rodents, receptor distribution can be strikingly diverse. Figure 3 shows the distribution of OXTR in the brains of rats, mice and voles. The species difference in expression is particularly robust in the NAc, where OXTR signaling facilitates pair bonding in prairie voles. Perhaps more remarkable is the individual variation in AVPR1A (Figure 4) and OXTR (Figure 5) distribution among individual prairie voles. This variation in receptor expression is functionally relevant and heritable, essential features of "evolvability."

A polymorphic variable repeat microsatellite in the 5 ' flanking region of the prairie vole Avprla, which is absent in the montane and meadow vole gene (Young et al., 1999), has been linked to individual variation in brain AVPR1A distribution and pair bonding behaviors in voles (Hammock \& Young, 2004, 2005). An elegant knock in mouse study demonstrated conclusively that sequence variation in this vole microsatellite was sufficient to alter brain receptor distribution and behavior in mice (Donaldson \& Young, 2013). Thus instability in microsatellite repetitive structure due to gene duplication slippage was proposed as an "evolutionary tuning knob" to generate diversity in receptor expression in the brain (Young \& Hammock, 

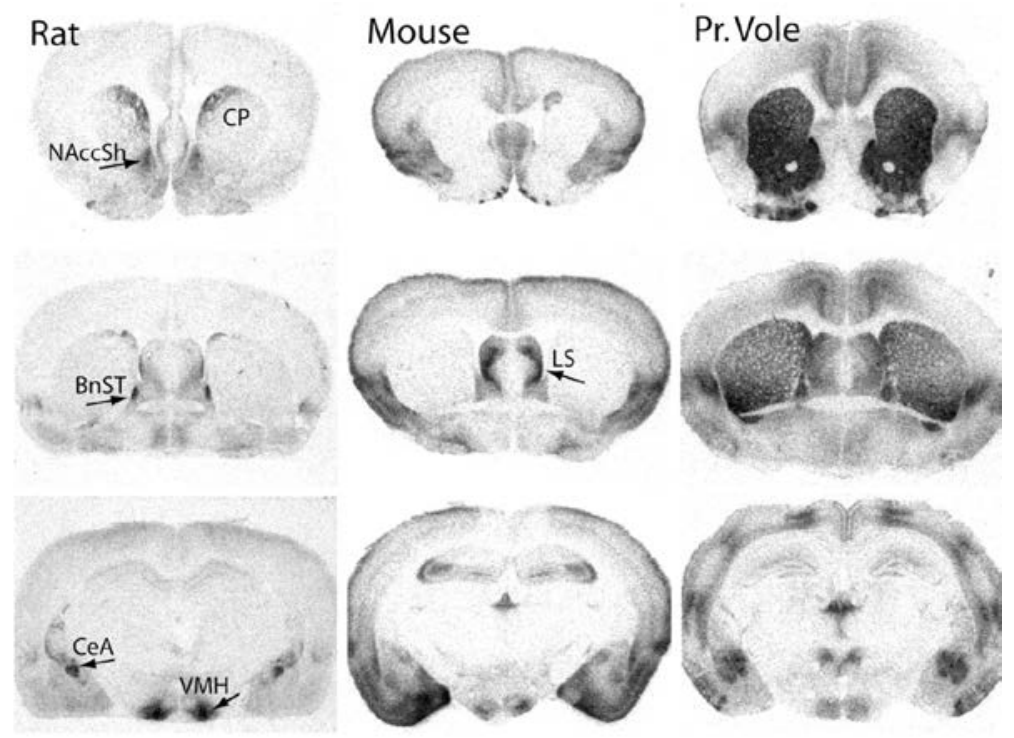

Figure 3. Receptor autoradiograms illustrating oxytocin receptor (OXT) binding sites in the forebrain of rat, mouse, and prairie vole. Receptor distribution is conserved in some regions, including the lateral septum (LS), bed nucleus of the stria terminalis (BnST), central nucleus of the amygdala (CeA), and ventromedial nucleus of hypothalamus $(\mathrm{VMH})$. There are marked species differences in dorsal caudate putamen (CP) and nucleus accumbens shell (NAccSh). Reprinted from (Burbach, Young, \& Russell, 2006).
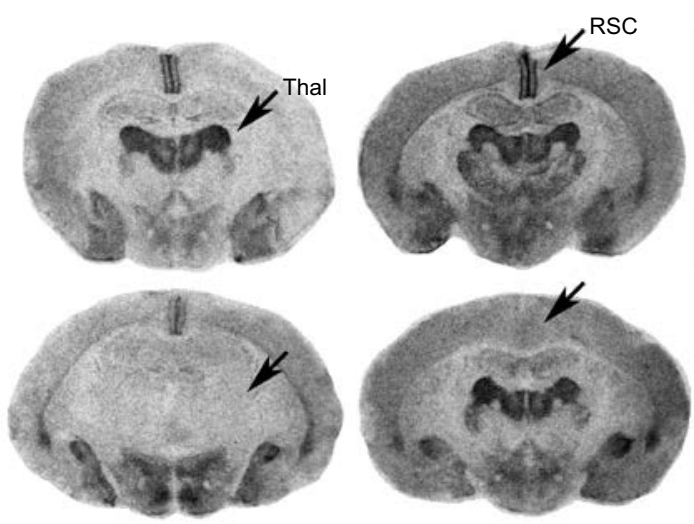

Figure 4. Receptor autoradiograms illustrating individual variation in $\mathrm{V} 1 \mathrm{a}$ vasopressin receptor (AVPR1A) binding in prairie voles. Note the robust differences in the Thal and $\mathrm{RSC}$, indicated by arrows. Thal, thalamus; RSC, retrosplenial cortex.

2007). Interestingly variation in a similar polymorphic microsatellite region in the AVPR1A of primates (Donaldson et al., 2008) is associated with pair bonding behavior in humans (Walum et al., 2008), and social joint attention (Hopkins et al., 2014) and other

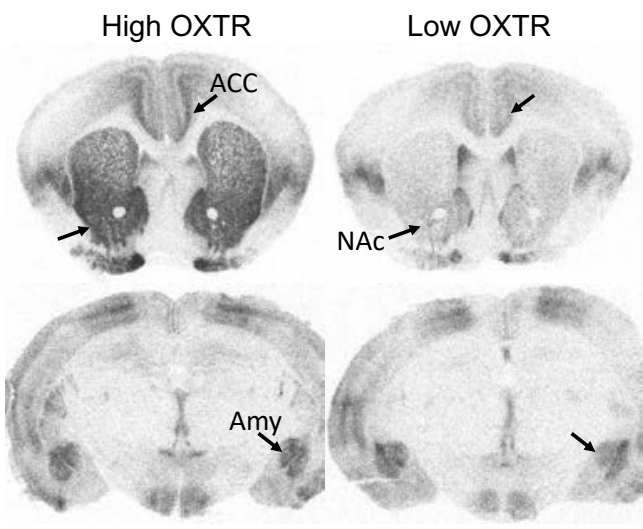

Figure 5. Receptor autoradiograms illustrating individual variation in oxytocin receptor (OXTR) binding in prairie voles. Note the robust variation in binding in the NAcc relative to Amy and other areas. ACC, anterior cingulate cortex; Amy, amygdala; NAcc, nucleus accumbens.

behavioral and brain traits in chimpanzees (Hopkins, Donaldson, \& Young, 2012; Latzman, Hopkins, Keebaugh, \& Young, 2014; Mahovetz, Young, \& Hopkins, 2016; Mulholland et al., 2020). Subsequent studies in voles have revealed other genetic 
variations throughout the Avprla gene that predict epigenetic status, AVPR1A expression in the retrosplenial cortex (RSC), and reproductive strategy and fitness in prairie voles (Okhovat et al., 2015). Thus, subtle DNA sequence variants can robustly affect AVPR1A distribution in the brain and behavior.

Variation in OXTR density in the NAc of prairie voles has been associated with individual variation in alloparental care (Olazabal \& Young, 2006), pair bonding (Ross, Freeman, et al., 2009), and susceptibility to early-life social deprivation (Barrett, Arambula, \& Young, 2015). This individual variation in OXTR density in the NAc is due to variation in gene transcription, is heritable, and is robustly predicted by a set of single nucleotide polymorphisms (SNPs) throughout the prairie vole Oxtr gene (King, Walum, Inoue, Eyrich, \& Young, 2016). Interestingly, OXTR density is not as variable in other brain regions, such as $\mathrm{PFC}$, amygdala or insular cortex, and the Oxtr SNPs do not correlated with expression in those areas (Figure $5)$. While each of the 15 SNPs that are in LD robustly predict $\sim 80 \%$ of the variation in Oxtr expression in NAc, it is not clear if one or any of these SNPs are functionally influencing expression. The sequencing techniques used thus far do not detect other common forms of sequence variation (e.g. indels or repetitive elements) and current research is attempting to identify the functional variants that cause the variation in expression.

Both Oxtr and Avprla show remarkable diversity in expression that is just as robust within species as it is across species. This variation in expression is genetic and thus heritable, and there are behavioral consequences of expression variation. Thus, Avpr1a and Oxtr represent clear examples of genes conferring "evolvability." The phenotypic plasticity of the expression profile of these receptor genes is in stark contrast to the ligand genes, Avp and $O x t$, which are expressed in a conserved neuroanatomical pattern in the hypothalamus across verte- brates, and even in homologous cell types in invertebrates. This phylogenetic stability in Avp/Oxt expression appears to be due to conserved transcriptional regulatory machinery across phyla. Neurosecretory cells producing AVP homologues in zebrafish and an annelid (segmented worm) express common transcription factors, micro-RNAs, and share embryonic migratory patterns (Tessmar-Raible et al., 2007). Furthermore, a BAC transgene comprising the puffer fish (Fugu rubripes) $O x t$ gene and approximately $16 \mathrm{~kb}$ of 5 ' flanking regulatory sequence expressed the Fugu Oxt transcript specifically in hypothalamic $O x t$ neurons in three different transgenic rat lines, independent of insertion site (Venkatesh, Si-Hoe, Murphy, \& Brenner, 1997). Similar transgenic experiments transferring $\sim 5 \mathrm{~kb}$ of the Fugu Oxt or $\sim 9 \mathrm{~kb}$ of the Avp resulted in expression of the fish genes in mouse $O x t$ and Avp neurons, respectively (Gilligan, Brenner, \& Venkatesh, 2003). This suggest there is remarkable functional conservation, even rigidity, of regulatory controls between the Fugu and rodent Oxt genes, despite being separate by 400 million years of evolution.

\section{Evolution of novel social behaviors}

As mentioned, much of biomedical neuroscience research is predicated on the assumption of conversation in brain structure and function between rodents and man. Of course, there must also be fundamental differences in brain organization that account for the remarkable diversity in behaviors and capacities across species. A fundamental question of evolutionary biology is how novel complex traits, such as monogamy or empathy, evolve from a mechanistic level? This is question is particularly perplexing given the apparent conservation in brain structure among mammals. Comparative studies, such as those described above in voles, hold promise for understanding how selective forces and random mutations in DNA sequence can give rise to diversity in behavior. Here we highlight two principles 
that have emerged from vole research that are hypotheses, or models that require testing empirically.

The first principle, that evolution "tweaks" pre-existing neural circuits/mechanisms to give rise to new behaviors, was perhaps anticipated by Charles Darwin in a passage in his Decent of Man, and Selection in Relation to Sex:

"Woman seems to differ from man in mental disposition, chiefly in her greater tenderness and less selfishness. ... Woman, owing to her maternal instincts, displays these qualities to her infants in an eminent degree; therefore it is likely that she would extend them towards her fellow creatures."

This explanation of sex differences in human empathetic responses, or compassion, reveals Darwin's intuition that the mechanisms driving maternal nurturing may not be rigidly constrained to offspring, but imprecision in those mechanisms may enhance empathy more broadly to unrelated conspecifics in individuals possessing a maternally tuned brain (i.e. women). Indeed, the observation that OXT, the quintessential maternal peptide, is critically involved in empathy-based consoling behavior toward non-kin in prairie voles, which does not occur in meadow voles, supports the principle related to Darwin's observation. We hypothesize that repurposing of neural mechanisms from one function to other functions represents a common mechanism by which complex traits such as pair bonding, selective aggression and empathy can rapidly evolve. We hypothesize that OXT-dependent circuit mechanisms involved in maternal nurturing and mother-infant bonding were "tweaked" evolutionarily to give rise to empathetic consoling and pair bonding, respectively (Figure 6). Likewise, the AVP-dependent circuits regulating territoriality (e.g. scent marking and territorial aggression) were "tweaked" to give rise to mate guarding and selective aggression (Freeman \& Young, 2013). Thus, maternal care, ubiquitous among mammals, is the evolutionary antecedent of the nurturing pair bond and empathetic consoling behavior found in monogamous species. By contrast, territorial behavior is the evolutionary antecedent of the possessive pair bond and mate guarding selective aggression. By extension, the OXTdependent neural mechanisms of pair
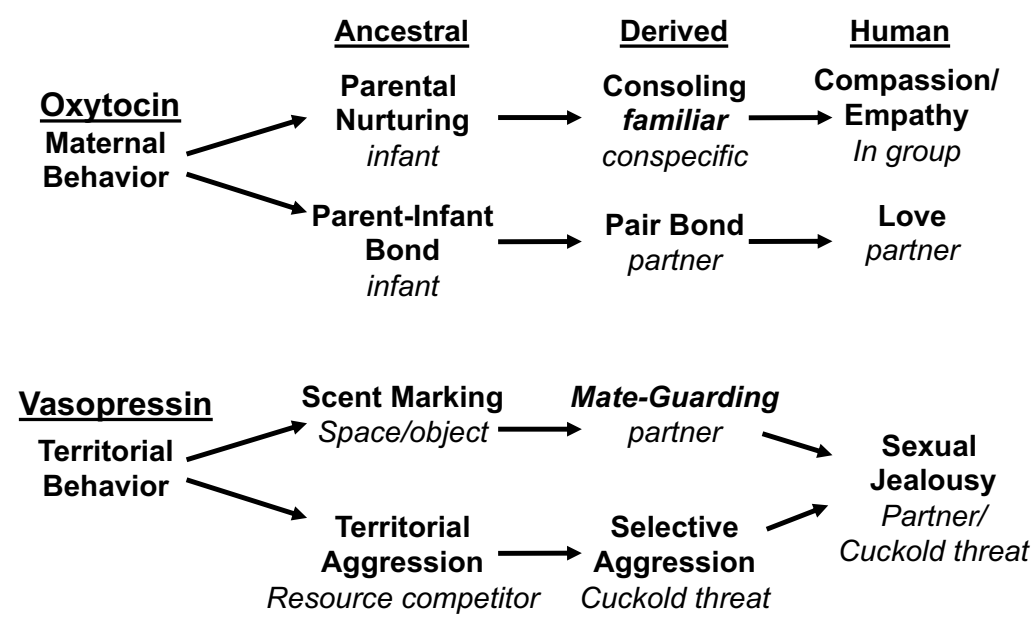

Figure 6. A model illustrating the repurposing of ancient circuits regulating maternal care and territorial behavior, modulated by oxytocin and vasopressin, to give rise to derived traits related to monogamy. Consoling, pair bonding, mate guarding and selective aggression are hypothesized to share deep homology with compassion, love and jealousy in humans. In this evolutionary process the subject which the behavior is directed to (in italics) shifts from infants to mates or conspecifics and from territory and resource competitor to partner and cuckhold treats. 
bonding and consoling behavior in animals, such as voles, are the evolutionary antecedents of love and compassion, while AVPdependent mate guarding and selective aggression in monogamous males are the antecedents of jealousy.

If this principle is correct, how did empathy evolve in males? Dulac, Kimchi and colleagues showed that the neural circuits governing male-typical sexual and parental behavior are fundamentally present in both males and females, but they are suppressed in one sex to generate robust sex differences in reproductive behavior (Kimchi, Xu, \& Dulac, 2007; Wu, Autry, Bergan, WatabeUchida, \& Dulac, 2014). Thus, it is likely that mechanism governing maternal nurturing, mother-infant bonding, and territorial behavior are likewise present in both sexes in all mammalian species. Changes in neuromodulatory mechanisms can loosen the "brakes" on those circuits and broaden their targets to give rise to pair bonding and consoling behaviors in both sexes directed toward mates or familiar conspecifics, rather than being restricted to pups. Mate guarding selective aggression and pair bonding in males are directed towards partners and sexual competitors rather than towards territory or resource competitors (Figure 6). This repurposing of ancient neural circuits is analogous to ancient architects who easily repurposed old structures for new purposes. For(Horn et al., 2020) example, in 1453 the Ottoman Empire constructed a magnificent Islamic grand mosque in Istanbul simply by modifying the preexisting Hagia Sophia cathedral originally constructed over 1000 years earlier by Emperor Constantine. Evolution appears to take similarly easy routes.

Alternative hypotheses for the evolution of prosocial behaviors have been proposed based on observations in primates and corvids (Horn et al., 2020). The cooperative breeding hypothesis proposes that prosocial behaviors (i.e., sharing food with non-kin) evolved from through expansion of psychological processes regulating alloparental care in cooperative breeding species. The colonial breeding, or self-domestication hypothesis postulates that reduced aggression and heightened social tolerance, such as required by colonial breeding in birds is a likely evolutionary pathway to prosocial behaviors. Empirical data from corvid species support both potential pathways with some evidence of interesting sex differences. These hypotheses, while rooted in a psychological rather than neural mechanism framework, are consistent with the notion that evolution "tweaks" pre-existing neural mechanisms to give rise to new behaviors.

The second principle is that certain genes encoding neuromodulators, such as Oxtr and Avprla possess an inherent instability in tissue specific transcription that generates diversity in expression among preexisting behavioral circuits in the brain. We hypothesize differential expression, arising initially as individual variation in receptor distribution, can differentially modulate circuits to allow cryptic behaviors to emerge in new contexts and directed to new targets (derived traits). Thus, maternal behavior morphs into pair bonding, consoling, love and compassion, while territorial behavior transitions to mate guarding and jealousy.

How is the evolutionary plasticity in neuropeptide receptor expression achieved? One possibility is that regulatory proteins driving brain region-specific expression in the brain are themselves variably expressed, leading to diversity in Oxtr and Avpr1a expression. However, the identification of subtle genetic variants that influence expression in these genes does not support that hypothesis. Alternatively, the Oxtr and Avprla genes may have rather nuanced, or fragile transcriptional regulation, such that subtle changes in gene sequence (SNPs) or structure (e.g. methylation, indels, variable repeats) can lead to diversity in expression pattern, altering neuromodulation of preexisting circuits, and giving rise to novel behavioral phenotypes upon which natural selection can act. If this is the case, errors in DNA replication or distal genomic landscape alterations (indels, translocations) can dra- 
matically alter the behavioral circuits modulated by OXT and AVP. Further studies on the effects of sequence variation and genomic landscape on receptor expression pattern are needed.

\section{Conclusions}

It is necessary to study diverse species in order to gain a fuller understanding of the neurobiological processes involved in behaviors relevant to human behaviors. It is important to adapt tools for circuit manipulations that are available in mice to those diverse model species. The OXT and AVP systems play important neuromodulatory roles on social behavioral circuits and changes in brain receptor distributions in those circuits likely underlies diversity in social behaviors. Subtle DNA sequence variation in Oxtr and Avprla seems to profoundly affect expression levels even within species. A major unanswered question with tremendous evolutionary implications is what gives the Oxtr and Avprla genes such flexibility, fragility, or evolvability. One possibility is that while the expression of many genes (e.g. $O x t$ ) are rigidly set by proximal regulatory sequences, Oxtr and Avprla are highly sensitive to subtle changes in sequence and chromosomal environment. While this hypothesis remains to be tested, we believe this transcriptional flexibility is key to the origin of diversity in social behavior, and enables rapid social behavioral adaptation through natural selection, and contributes to the remarkable diversity in social and reproductive behaviors in the animal kingdom.

\section{Acknowledgments}

Preparation of this manuscript was supported by funding from the International Education and Research Laboratory Program of University of Tsukuba, which supports the authors' contribution and the Center for Social Neural Networks.

\section{References}

Albers, H. E. (2012). The regulation of social recognition, social communication and aggression: vasopressin in the social behavior neural network. Horm Behav, 61(3), 283-292. doi:10.1016/j.yhbeh.2011.10.007

Amadei, E. A., Johnson, Z. V., Jun Kwon, Y., Shpiner, A. C., Saravanan, V., Mays, W. D., ... Liu, R. C. (2017). Dynamic corticostriatal activity biases social bonding in monogamous female prairie voles. Nature, 546(7657), 297-301. doi:10.1038/nature22381

Barrett, C. E., Arambula, S. E., \& Young, L. J. (2015). The oxytocin system promotes resilience to the effects of neonatal isolation on adult social attachment in female prairie voles. Transl Psychiatry, 5, e606. doi:10.1038/tp.2015.73

Barrett, C. E., Keebaugh, A. C., Ahern, T. H., Bass, C. E., Terwilliger, E. F., \& Young, L. J. (2013). Variation in vasopressin receptor (Avprla) expression creates diversity in behaviors related to monogamy in prairie voles. Horm Behav, 63(3), 518-526. doi:10.1016/j.yhbeh.2013.01.005

Bethlehem, R. A. I., Lombardo, M. V., Lai, M. C., Auyeung, B., Crockford, S. K., Deakin, J., ... Baron-Cohen, S. (2017). Intranasal oxytocin enhances intrinsic corticostriatal functional connectivity in women. Transl Psychiatry, 7(4), e1099. doi:10.1038/tp.2017.72

Blumstein, D. T., Ebensperger, L. A., Hayes, L. D., Vasquez, R. A., Ahern, T. H., Burger, J. R., ... Young, L. J. (2010). Toward an integrative understanding of social behavior: new models and new opportunities. Front Behav Neurosci, 4, 34. doi:10.3389/fnbeh.2010.00034

Boender, A. J., \& Young, L. J. (2020). Oxytocin, vasopressin and social behavior in the age of genome editing: A comparative perspective. Horm Behav, 124, 104780. doi:10.1016/j.yhbeh.2020.104780

Bosch, O. J., Dabrowska, J., Modi, M. E., Johnson, Z. V., Keebaugh, A. C., Barrett, C. E., ... Young, L. J. (2016). Oxytocin in the nucleus accumbens shell reverses CRFR2- 
evoked passive stress-coping after partner loss in monogamous male prairie voles. Psychoneuroendocrinology, 64, 66-78. doi:10.1016/j.psyneuen.2015.11.011

Bosch, O. J., Nair, H. P., Ahern, T. H., Neumann, I. D., \& Young, L. J. (2009). The CRF system mediates increased passive stress-coping behavior following the loss of a bonded partner in a monogamous rodent. Neuropsychopharmacology, 34(6), 1406-1415. doi:10.1038/npp.2008.154

Bosch, O. J., Pohl, T. T., Neumann, I. D., \& Young, L. J. (2018). Abandoned prairie vole mothers show normal maternal care but altered emotionality: Potential influence of the brain corticotropin-releasing factor system. Behav Brain Res, 341, 114121. doi:10.1016/j.bbr.2017.12.034

Bosch, O. J., \& Young, L. J. (2018). Oxytocin and Social Relationships: From Attachment to Bond Disruption. Curr Top Behav Neurosci, 35, 97-117. doi:10.1007/7854_2017_10

Burbach, P., Young, L. J., \& Russell, J. (2006). Oxytocin: Synthesis, Secretion and Reproductive Functions. In J. D. Neill (Ed.), Knobil an Neill's Physiology of Reproduction. (Third ed., pp.3055-3127): Elsevier.

Burkett, J. P., Andari, E., Johnson, Z. V., Curry, D. C., de Waal, F. B., \& Young, L. J. (2016). Oxytocin-dependent consolation behavior in rodents. Science, 351(6271), 375-378. doi:10.1126/science.aac4785

Burkett, J. P., \& Young, L. J. (2012). The behavioral, anatomical and pharmacological parallels between social attachment, love and addiction. Psychopharmacology (Berl), 224(1), 1-26. doi:10.1007/s00213-012-2794-x

DeMayo, M. M., Young, L. J., Hickie, I. B., Song, Y. J. C., \& Guastella, A. J. (2019). Circuits for social learning: A unified model and application to Autism Spectrum Disorder. Neurosci Biobehav Rev, 107, 388-398. doi:10.1016/j.neubiorev.2019.09.034

Dolen, G., Darvishzadeh, A., Huang, K. W., \& Malenka, R. C. (2013). Social reward requires coordinated activity of nucleus accumbens oxytocin and serotonin. Nature, 501(7466), 179-184.

doi:10.1038/nature12518

Donaldson, Z. R., Kondrashov, F. A., Putnam, A., Bai, Y., Stoinski, T. L., Hammock, E. A., \& Young, L. J. (2008). Evolution of a behavior-linked microsatellite-containing element in the 5' flanking region of the primate AVPR1A gene. BMC Evol Biol, 8, 180. doi:10.1186/1471-2148-8-180

Donaldson, Z. R., Spiegel, L., \& Young, L. J. (2010). Central vasopressin V1a receptor activation is independently necessary for both partner preference formation and expression in socially monogamous male prairie voles. Behav Neurosci, 124(1), 159163. doi:10.1037/a0018094

Donaldson, Z. R., Yang, S. H., Chan, A. W., \& Young, L. J. (2009). Production of germline transgenic prairie voles (Microtus ochrogaster) using lentiviral vectors. Biol Reprod, 81(6), 1189-1195. doi:10.1095/biolreprod.109.077529

Donaldson, Z. R., \& Young, L. J. (2008). Oxytocin, vasopressin, and the neurogenetics of sociality. Science, 322(5903), 900904. doi:10.1126/science.1158668

Donaldson, Z. R., \& Young, L. J. (2013). The relative contribution of proximal 5 flanking sequence and microsatellite variation on brain vasopressin 1a receptor (Avprla) gene expression and behavior. PLoS Genet, 9(8), e1003729. doi:10.1371/journal.pgen.1003729

Ferguson, J. N., Aldag, J. M., Insel, T. R., \& Young, L. J. (2001). Oxytocin in the medial amygdala is essential for social recognition in the mouse. $J$ Neurosci, 21(20), 8278-8285.

Fink, S., Excoffier, L., \& Heckel, G. (2006). Mammalian monogamy is not controlled by a single gene. Proc Natl Acad Sci U S A, 103(29), 10956-10960. doi:10.1073/pnas.0602380103

Ford, C. L., \& Young, L. J. (2020). Translational opportunities for circuitbased social neuroscience: advancing 21st century psychiatry. Curr Opin Neurobiol, 68, 1-8. doi:10.1016/j.conb.2020.11.007 
Freeman, S. M., Inoue, K., Smith, A. L., Goodman, M. M., \& Young, L. J. (2014). The neuroanatomical distribution of oxytocin receptor binding and mRNA in the male rhesus macaque (Macaca mulatta). Psychoneuroendocrinology, 45, 128-141. doi:10.1016/j.psyneuen.2014.03.023

Freeman, S. M., Walum, H., Inoue, K., Smith, A. L., Goodman, M. M., Bales, K. L., \& Young, L. J. (2014). Neuroanatomical distribution of oxytocin and vasopressin 1a receptors in the socially monogamous coppery titi monkey (Callicebus cupreus). Neuroscience, 273, 12-23. doi:10.1016/j.neuroscience.2014.04.055

Freeman, S. M., \& Young, L. J. (2013). Oxytocin, vasopressin, and the evolution of mating systems in mammals. Oxytocin, Vasopressin and Related Peptides in the Regulation of Behavior, 128-147. doi:Book Doi 10.1017/Cbo9781139017855

Freeman, S. M., \& Young, L. J. (2016). Comparative Perspectives on Oxytocin and Vasopressin Receptor Research in Rodents and Primates: Translational Implications. J Neuroendocrinol, 28(4). doi:10.1111/jne.12382

Froemke, R. C., \& Young, L. J. (2021). Oxytocin, neural plasticity, and social behavior. Annu. Rev. Neurosci., 44, 359381.

doi:10.1146/annurev-neuro-102320-102847

Gilligan, P., Brenner, S., \& Venkatesh, B. (2003). Neurone-specific expression and regulation of the pufferfish isotocin and vasotocin genes in transgenic mice. $J$ Neuroendocrinol, 15(11), 1027-1036. doi:10.1046/j.1365-2826.2003.01090.x

Gobrogge, K. L., Liu, Y., Young, L. J., \& Wang, Z. (2009). Anterior hypothalamic vasopressin regulates pair-bonding and drug-induced aggression in a monogamous rodent. Proc Natl Acad Sci U S A, 106(45), 19144-19149. doi:10.1073/pnas.0908620106

Goodson, J. L., Schrock, S. E., Klatt, J. D., Kabelik, D., \& Kingsbury, M. A. (2009). Mesotocin and nonapeptide receptors promote estrildid flocking behavior.
Science, 325(5942), 862-866.

doi:10.1126/science.1174929

Grebe, N. M., Sharma, A., Freeman, S. M., Palumbo, M. C., Patisaul, H. B., Bales, K. L., \& Drea, C. M. (2021). Neural correlates of mating system diversity: oxytocin and vasopressin receptor distributions in monogamous and non-monogamous Eulemur. Sci Rep, 11(1), 3746. doi:10.1038/s41598-021-83342-6

Grinevich, V., Knobloch-Bollmann, H. S., Eliava, M., Busnelli, M., \& Chini, B. (2016). Assembling the Puzzle: Pathways of Oxytocin Signaling in the Brain. Biol Psychiatry, 79(3), 155-164. doi:10.1016/j.biopsych.2015.04.013

Hammock, E. A., \& Young, L. J. (2004). Functional microsatellite polymorphism associated with divergent social structure in vole species. Mol Biol Evol, 21(6), 10571063. doi:10.1093/molbev/msh104

Hammock, E. A., \& Young, L. J. (2005). Microsatellite instability generates diversity in brain and sociobehavioral traits. Science, 308(5728), 1630-1634. doi:10.1126/science.1111427

Hofmann, H. A., Beery, A. K., Blumstein, D. T., Couzin, I. D., Earley, R. L., Hayes, L. D., ... Rubenstein, D. R. (2014). An evolutionary framework for studying mechanisms of social behavior. Trends Ecol Evol, 29(10), 581-589. doi:10.1016/j.tree.2014.07.008

Hopkins, W. D., Donaldson, Z. R., \& Young, L. J. (2012). A polymorphic indel containing the RS3 microsatellite in the 5' flanking region of the vasopressin V1a receptor gene is associated with chimpanzee (Pan troglodytes) personality. Genes Brain Behav, 11(5), 552-558. doi:10.1111/j.1601-183X.2012.00799.x

Hopkins, W. D., Keebaugh, A. C., Reamer, L. A., Schaeffer, J., Schapiro, S. J., \& Young, L. J. (2014). Genetic influences on receptive joint attention in chimpanzees (Pan troglodytes). Sci Rep, 4, 3774. doi:10.1038/srep03774

Horie, K., Inoue, K., Nishimori, K., \& Young, L. J. (2020). Investigation of Oxtr-expressing Neurons Projecting to Nucleus Accum- 
bens using Oxtr-ires-Cre Knock-in prairie Voles (Microtus ochrogaster). Neuroscience, 448, 312-324. doi:10.1016/j.neuroscience.2020.08.023

Horie, K., Inoue, K., Suzuki, S., Adachi, S., Yada, S., Hirayama, T., ... Nishimori, K. (2019). Oxytocin receptor knockout prairie voles generated by CRISPR/Cas9 editing show reduced preference for social novelty and exaggerated repetitive behaviors. Horm Behav, 111, 60-69. doi:10.1016/j.yhbeh.2018.10.011

Horn, L., Bugnyar, T., Griesser, M., Hengl, M., Izawa, E. I., Oortwijn, T., ... Massen, J. J. (2020). Sex-specific effects of cooperative breeding and colonial nesting on prosociality in corvids. Elife, 9. doi:10.7554/eLife.58139

Johnson, Z. V., Walum, H., Jamal, Y. A., Xiao, Y., Keebaugh, A. C., Inoue, K., \& Young, L. J. (2016). Central oxytocin receptors mediate mating-induced partner preferences and enhance correlated activation across forebrain nuclei in male prairie voles. Horm Behav, 79, 8-17. doi:10.1016/j.yhbeh.2015.11.011

Johnson, Z. V., \& Young, L. J. (2017). Oxytocin and vasopressin neural networks: Implications for social behavioral diversity and translational neuroscience. Neurosci Biobehav Rev, 76(Pt A), 87-98. doi:10.1016/j.neubiorev.2017.01.034

Johnson, Z. V., \& Young, L. J. (2018). Evolutionary diversity as a catalyst for biological discovery. Integr Zool, 13(6), 616-633. doi:10.1111/1749-4877.12339

Keebaugh, A. C., Barrett, C. E., Laprairie, J. L., Jenkins, J. J., \& Young, L. J. (2015). RNAi knockdown of oxytocin receptor in the nucleus accumbens inhibits social attachment and parental care in monogamous female prairie voles. Soc Neurosci, 10(5), 561-570. doi:10.1080/17470919.2015.1040893

Keebaugh, A. C., \& Young, L. J. (2011). Increasing oxytocin receptor expression in the nucleus accumbens of pre-pubertal female prairie voles enhances alloparental responsiveness and partner preference formation as adults. Hormones and Behavior, 60(5), 498-504. doi:10.1016/j.yhbeh.2011.07.018

Kimchi, T., Xu, J., \& Dulac, C. (2007). A functional circuit underlying male sexual behaviour in the female mouse brain. Nature, 448(7157), 1009-1014. doi:10.1038/nature06089

King, L. B., Walum, H., Inoue, K., Eyrich, N. W., \& Young, L. J. (2016). Variation in the Oxytocin Receptor Gene Predicts Brain Region-Specific Expression and Social Attachment. Biol Psychiatry, 80(2), 160169. doi:10.1016/j.biopsych.2015.12.008

King, L. B., \& Young, L. J. (2016). Oxytocin, Vasopressin and Diversity in Social Behavior. In D. Murphy \& H. Gainer (Eds.), Molecular Neuroendocrinology: From Genome to Physiology: John Wiley \& Sons, Ltd.

Latzman, R. D., Hopkins, W. D., Keebaugh, A. C., \& Young, L. J. (2014). Personality in chimpanzees (Pan troglodytes): exploring the hierarchical structure and associations with the vasopressin V1A receptor gene. PLoS One, 9(4), e95741.

doi:10.1371/journal.pone.0095741

Lim, M. M., Murphy, A. Z., \& Young, L. J. (2004). Ventral striatopallidal oxytocin and vasopressin V1a receptors in the monogamous prairie vole (Microtus ochrogaster). J Comp Neurol, 468(4), 555570. doi:10.1002/cne.10973

Lim, M. M., Wang, Z., Olazabal, D. E., Ren, X., Terwilliger, E. F., \& Young, L. J. (2004). Enhanced partner preference in a promiscuous species by manipulating the expression of a single gene. Nature, 429(6993), 754-757. doi:10.1038/nature02539

Lim, M. M., \& Young, L. J. (2004). Vasopressindependent neural circuits underlying pair bond formation in the monogamous prairie vole. Neuroscience, 125(1), 35-45. doi:10.1016/j.neuroscience.2003.12.008

Lischinsky, J. E., \& Lin, D. (2020). Neural mechanisms of aggression across species. Nat Neurosci, 23(11), 1317-1328. doi:10.1038/s41593-020-00715-2

Mahovetz, L. M., Young, L. J., \& Hopkins, 
W. D. (2016). The influence of AVPR1A genotype on individual differences in behaviors during a mirror self-recognition task in chimpanzees (Pan troglodytes). Genes Brain Behav, 15(5), 445-452. doi:10.1111/gbb.12291

McGraw, L. A., \& Young, L. J. (2010). The prairie vole: an emerging model organism for understanding the social brain. Trends Neurosci, 33(2), 103-109. doi:10.1016/j.tins.2009.11.006

Mulholland, M. M., Navabpour, S. V., Mareno, M. C., Schapiro, S. J., Young, L. J., \& Hopkins, W. D. (2020). AVPR1A variation is linked to gray matter covariation in the social brain network of chimpanzees. Genes Brain Behav, 19(4), e12631. doi:10.1111/gbb.12631

Nair, H. P., \& Young, L. J. (2006). Vasopressin and pair-bond formation: genes to brain to behavior. Physiology (Bethesda), 21, 146-152. doi:10.1152/physiol.00049.2005

Nardou, R., Lewis, E. M., Rothhaas, R., Xu, R., Yang, A., Boyden, E., \& Dolen, G. (2019). Oxytocin-dependent reopening of a social reward learning critical period with MDMA. Nature, 569(7754), 116-120. doi:10.1038/s41586-019-1075-9

Oettl, L. L., Ravi, N., Schneider, M., Scheller, M. F., Schneider, P., Mitre, M., ... Kelsch, W. (2016). Oxytocin Enhances Social Recognition by Modulating Cortical Control of Early Olfactory Processing. Neuron, 90(3), 609-621. doi:10.1016/j.neuron.2016.03.033

Okhovat, M., Berrio, A., Wallace, G., Ophir, A. G., \& Phelps, S. M. (2015). Sexual fidelity trade-offs promote regulatory variation in the prairie vole brain. Science, 350(6266), 1371-1374. doi:10.1126/science.aac5791

Olazabal, D. E., \& Young, L. J. (2006). Oxytocin receptors in the nucleus accumbens facilitate "spontaneous" maternal behavior in adult female prairie voles. Neuroscience, 141(2), 559-568. doi:10.1016/j.neuroscience.2006.04.017

Payne, J. L., \& Wagner, A. (2019). The causes of evolvability and their evolution. Nat Rev Genet, 20(1), 24-38.
doi:10.1038/s41576-018-0069-Z

Pitkow, L. J., Sharer, C. A., Ren, X., Insel, T. R., Terwilliger, E. F., \& Young, L. J. (2001). Facilitation of affiliation and pairbond formation by vasopressin receptor gene transfer into the ventral forebrain of a monogamous vole. J Neurosci, 21(18), 7392-7396.

Pohl, T. T., Young, L. J., \& Bosch, O. J. (2019). Lost connections: Oxytocin and the neural, physiological, and behavioral consequences of disrupted relationships. Int J Psychophysiol, 136, 54-63. doi:10.1016/j.ijpsycho.2017.12.011

Rogers Flattery, C. N., Coppeto, D. J., Inoue, K., Rilling, J. K., Preuss, T. M., \& Young, L. J. (2021). Distribution of brain oxytocin and vasopressin V1a receptors in chimpanzees (Pan troglodytes): Comparison with humans and other primate species. Submitted.

Ross, H. E., Cole, C. D., Smith, Y., Neumann, I. D., Landgraf, R., Murphy, A. Z., \& Young, L. J. (2009). Characterization of the oxytocin system regulating affiliative behavior in female prairie voles. Neuroscience, 162(4), 892-903. doi:10.1016/j.neuroscience.2009.05.055

Ross, H. E., Freeman, S. M., Spiegel, L. L., Ren, X., Terwilliger, E. F., \& Young, L. J. (2009). Variation in oxytocin receptor density in the nucleus accumbens has differential effects on affiliative behaviors in monogamous and polygamous voles. $J$ Neurosci, 29(5), 1312-1318. doi:10.1523/JNEUROSCI.5039-08.2009

Ross, H. E., \& Young, L. J. (2009). Oxytocin and the neural mechanisms regulating social cognition and affiliative behavior. Front Neuroendocrinol, 30(4), 534-547. doi:10.1016/j.yfrne.2009.05.004

Scheele, D., Wille, A., Kendrick, K. M., Stoffel-Wagner, B., Becker, B., Gunturkun, O., ... Hurlemann, R. (2013). Oxytocin enhances brain reward system responses in men viewing the face of their female partner. Proc Natl Acad Sci U S A, 110(50), 20308-20313. doi:10.1073/pnas.1314190110 
Scribner, J. L., Vance, E. A., Protter, D. S. W., Sheeran, W. M., Saslow, E., Cameron, R. T., ... Donaldson, Z. R. (2020). A neuronal signature for monogamous reunion. Proc Natl Acad Sci U S A, 117(20), 11076-11084. doi:10.1073/pnas.1917287117

Tessmar-Raible, K., Raible, F., Christodoulou, F., Guy, K., Rembold, M., Hausen, H., \& Arendt, D. (2007). Conserved sensoryneurosecretory cell types in annelid and fish forebrain: insights into hypothalamus evolution. Cell, 129(7), 1389-1400. doi:10.1016/j.cell.2007.04.041

Venkatesh, B., Si-Hoe, S. L., Murphy, D., \& Brenner, S. (1997). Transgenic rats reveal functional conservation of regulatory controls between the Fugu isotocin and rat oxytocin genes. Proc Natl Acad Sci U S A, 94(23), 12462-12466. doi:10.1073/pnas.94.23.12462

Walum, H., Westberg, L., Henningsson, S., Neiderhiser, J. M., Reiss, D., Igl, W., ... Lichtenstein, P. (2008). Genetic variation in the vasopressin receptor 1a gene (AVPR1A) associates with pair-bonding behavior in humans. Proc Natl Acad Sci U S A, 105(37), 14153-14156. doi:10.1073/pnas.0803081105

Walum, H., \& Young, L. J. (2018). The neural mechanisms and circuitry of the pair bond. Nat Rev Neurosci, 19(11), 643-654. doi:10.1038/s41583-018-0072-6

Wu, Z., Autry, A. E., Bergan, J. F., WatabeUchida, M., \& Dulac, C. G. (2014). Galanin neurons in the medial preoptic area govern parental behaviour. Nature, 509(7500), 325-330. doi:10.1038/nature13307

Young, L., \& Alexander, B. (2012). The Chemistry Between Us: Love, Sex and the Science of Attraction. New York, NY: Current.

Young, L. J. (1999). Frank A. Beach Award.
Oxytocin and vasopressin receptors and species-typical social behaviors. Horm Behav, 36(3), 212-221. doi:10.1006/hbeh.1999.1548

Young, L. J. (2009). Being human: love: neuroscience reveals all. Nature, 457(7226), 148. doi:10.1038/457148a

Young, L. J., \& Crews, D. (1995). Comparative neuroendocrinology of steroid receptor gene expression and regulation: Relationship to physiology and behavior. Trends Endocrinol Metab, 6(9-10), 317-323. doi:10.1016/1043-2760(95)00175-1

Young, L. J., \& Hammock, E. A. (2007). On switches and knobs, microsatellites and monogamy. Trends Genet, 23(5), 209-212. doi:10.1016/j.tig.2007.02.010

Young, L. J., Lim, M. M., Gingrich, B., \& Insel, T. R. (2001). Cellular mechanisms of social attachment. Horm Behav, 40(2), 133-138. doi:10.1006/hbeh.2001.1691

Young, L. J., Murphy Young, A. Z., \& Hammock, E. A. (2005). Anatomy and neurochemistry of the pair bond. J Comp Neurol, 493(1), 51-57. doi:10.1002/cne.20771

Young, L. J., Nilsen, R., Waymire, K. G., MacGregor, G. R., \& Insel, T. R. (1999). Increased affiliative response to vasopressin in mice expressing the V1a receptor from a monogamous vole. Nature, 400(6746), 766-768. doi:10.1038/23475

Young, L. J., Pitkow, L. J., \& Ferguson, J. N. (2002). Neuropeptides and social behavior: animal models relevant to autism. Mol Psychiatry, 7 Suppl 2, S38-39. doi:10.1038/sj.mp.4001175

Young, L. J., \& Wang, Z. (2004). The neurobiology of pair bonding. Nat Neurosci, 7(10), 1048-1054. doi:10.1038/nn1327

(2021. 4.2 受稿, 2021. 4.11 受理) 